\title{
Using the Ideas Café to Explore Trust in Autonomous Vehicles
}

\author{
Arun Ulahannan $^{1(凶)}$, Rebecca Cain ${ }^{2}$, Gunwant Dhadyalla ${ }^{1}$, \\ Paul Jennings ${ }^{1}$, Stewart Birrell ${ }^{1}$, Mike Waters ${ }^{3}$, and Alex Mouzakitis ${ }^{4}$ \\ ${ }^{1}$ WMG, University of Warwick, Coventry CV4 7ES, UK \\ \{A. Ulahannan, G. Dhadyalla, Paul. Jennings, \\ S.Birrel1\}@warwick.ac.uk \\ ${ }^{2}$ Loughborough University, Loughborough LE11 3TU, UK \\ R.Cain@1boro.ac.uk \\ ${ }^{3}$ Coventry University, Coventry CV1 5FB, UK \\ Mike.Waters@tfwm.org.uk \\ 4 Jaguar Land Rover, Abbey Road, Whitley, Coventry CV3 4LF, UK \\ amouzakl@jaguarlandrover.com
}

\begin{abstract}
Trust has been shown to play a key role in our ability to safely use autonomous vehicles; hence the authors used the Ideas Café to explore the factors affecting trust in autonomous vehicles. The Ideas Café is an informal collaborative event that brings the public together with domain experts for exploratory research. The authors structured the event around factors affecting trust in the technology, privacy and societal impact. The event followed a mixed methods approach using: table discussions, spectrum lines and line ups. 36 participants attended the Ideas Café event held at the Coventry Transport Museum in June 2017. Table discussions provided the key findings for Thematic Analysis as part of Grounded Theory; which found, contrary to current research trends, designing for the technology's integration with society as equally important for trust as the vehicle design itself. The authors also reported on the emergent high level interface guidelines.
\end{abstract}

Keywords: Human factors $\cdot$ Trust $\cdot$ Autonomous vehicles $\cdot$ Engagement Ideas Café

\section{Introduction}

An 'Ideas Café' is an event where members of the public are brought together with experts in the field around a particular topic, to stimulate conversation between the two. The format bears similarities to a café, with participants sat around tables with coffee and cake to engage in discussion. Through facilitation, the Ideas Café affords design researchers the opportunity to explore various experimental methods, as the authors demonstrate in this paper.

The format has been increasingly used by governments to garner consensus and build trust in a new idea or topic [1-3]. The Ideas Cafe enables futures thinking which has been shown to help people to envision the world they wish to live in, despite the 
uncertainty of the future $[4,5]$. Engaging with the public has been shown to be key to enabling society to successfully adapt to change [6], by enabling the sharing of knowledge and exposure to different viewpoints in a calm, supportive environment [7]. Autonomous vehicles are very much a reality; and it is essential that people can safely use this new technology as these vehicle become increasingly responsible for the safety of its occupants [8]. There is evidence to suggest that automated systems can be dangerous if the driver's relationship with the vehicle is suboptimal $[9,10]$.

Trust has been shown to be a major factor in this relationship and it is widely agreed that negative consequences occur as a result of the inappropriate level of trust placed in the system (both too much and too little trust) [11-13]. There is a pressing need to better understand how these automated systems, like autonomous vehicles, are designed [14].

However many factors contribute to trust development between the user and the autonomous system $[11,15,16]$. There have been attempts to quantify trust by looking at driver distraction and performance $[15,17,18]$. There are also qualitative methods used frequently in literature through interviews to determine the dimensions of trust [19-21]. Given the aforementioned futures thinking nature of the topic and the need to better understand what affects how trust is developed, there was an opportunity to explore trust on a broader scale and stimulate a discussion with the public. This paper describes the exploration of the public's perceptions of trust in autonomous vehicles using an Ideas Café public engagement event.

\section{Method}

The Ideas Cafes does not have a formal methodology associated with it, hence the authors explored how to best design the Ideas Café using a mixed methods approach (Table 1). Ethical approval was granted by Coventry University for the study P52764 Trust in Connected and Autonomous Vehicles: Ideas Café.

Table 1. Comparison of the KCP model and the principles of the Ideas Café [22-24]

\begin{tabular}{l|l|l}
\hline $\begin{array}{l}\text { Three success factors for } \\
\text { Ideas Cafes [22] }\end{array}$ & Key principles of an Ideas Café [23] & $\begin{array}{l}\text { KCP model } \\
{[24]}\end{array}$ \\
\hline Communication & Set the context & Knowledge \\
\hline Consultation & $\begin{array}{l}\text { Create a hospitable space } \\
\text { Explore questions that matter }\end{array}$ & $\begin{array}{l}\text { Cross-pollinate and connect diverse } \\
\text { perspectives }\end{array}$ \\
\cline { 2 - 3 } & $\begin{array}{l}\text { Listen together for patterns, insights and } \\
\text { deeper questions }\end{array}$ & \\
\hline Participation & Harvest and share collective discoveries & Proposal \\
\hline
\end{tabular}




\subsection{Event Design}

Three models of success for engagement methodologies were used in order to drive the design of the event [22-24]. It was evident that across the literature, the majority of the principles of an Ideas Café were centered around facilitating conceptualization and consultation, making it an ideal tool to explore the issues of trust in autonomous vehicles. The authors aimed to achieve the principles set out in Table 1 .

\subsection{Location and Personnel}

The Coventry Transport Museum provided a central location for participants and was booked for the 30th June 2017. Circular tables were setup in a cabaret formation to accommodate ten participants on each. Environmental cues can be influential in the participants' ability to engage with the task [25], so the selection of the venue was very important to the design of the Ideas Café to ensure an informal environment that encouraged participation.

The authors were assisted by ten table facilitators and a compere. Facilitators were given guidance on how to structure the hour given for the table discussions. Facilitators were encouraged to engage in the conversation as a participant (to achieve a two way flow of information), and to be wary of participants who felt uninvolved and to provide a platform for disagreement. The event day was hosted by a compere who was experienced working in similar Ideas Café events. The compere's role was independent of the research, which allowed the researchers to focus on the content of what was being said. The event was photographed by the University of Warwick photographer.

\subsection{Participants and Recruitment}

36 participants arrived on the day for the event ( 22 male, 11 female, 2 preferred not to say), recruited through opportunity sampling (Table 2). The age demographic can be seen in Table 2. Participants were split across 9 tables, with 4-7 participants and a one facilitator per table. From participant feedback, $44 \%$ of participants said they were 'extremely satisfied' with the event location, and 38\% said they were 'satisfied'. 100\% of participants said they were happy to be contacted again to take part in future research in the area, further suggesting that the Ideas Café was very successful.

Table 2. Age demographics (ordered by most populous categories)

\begin{tabular}{l|l}
\hline Age range & Number of participants \\
\hline $35-44$ & 9 \\
\hline $45-54$ & 9 \\
\hline $25-34$ & 5 \\
\hline $55-64$ & 5 \\
\hline 75 or older & 4 \\
\hline $18-24$ & 2 \\
\hline $65-74$ & 1 \\
\hline
\end{tabular}


The Ideas Cafe was advertised through social media, physical posters and newspaper articles with the help of the communications department at WMG, University of Warwick. The representation of society in the event can be seen below in Table 3. An opportune showing of a television documentary on autonomous cars meant many participants came prepared with discussion points, highlighting the importance of preeducation before the event.

Table 3. Representation of civil society at the event (ordered by most populous category)

\begin{tabular}{l|l}
\hline Occupation & Number of participants \\
\hline Retired & 9 \\
\hline Academia & 6 \\
\hline Student & 5 \\
\hline Engineering & 5 \\
\hline Government & 4 \\
\hline Unknown & 4 \\
\hline Charitable & 1 \\
\hline Publishing & 1 \\
\hline Marketing & 1 \\
\hline
\end{tabular}

\subsection{Spectrum Line}

A spectrum line (otherwise known as a semantic scale) was placed on a wall near the entrance of the venue. The intention was to see if there was any effect on participants' perception of autonomous vehicles before and after the event. This consisted of a line approximately $5 \mathrm{~m}$ long with a question placed above it. As participants entered, they were asked to stick their paper marker on the line in response to the question "Do you think driverless cars are a good idea?". This was also repeated at the end of the event to capture any differences in opinion from the start and end of the event. The line had no markings, similar to Visual Analogue Scales (VAS) which has been shown to be less prone to bias [26].

\section{$2.5 \quad$ Line up}

To counter participants potentially becoming restless after sitting for more than an hour, a standing physical spectrum line was used. Participants were asked to physically stand on a line on the floor to represent their opinion. The compere would then ask participants to explain their viewpoint to the group and encouraged those with opposing views to engage in the conversation. Further, this helped cross-pollinate ideas and bring together people with opposing viewpoints in conversation, as recommended by the guidelines in Table 1. However, results were not collected for this as its purpose was as more of an energizer. 


\subsection{Table Session}

Three areas of trust from literature were chosen for the table topics, these were: Trust in Technology, Privacy and Societal Impact. The authors created a set of bespoke artificial newspaper articles designed to help stimulate conversation around the table.

Participants were asked to capture their ideas on post-it notes and stick everything they produced onto a larger sheet of A2 paper. The authors did not provide any specific instructions on how participants should build or structure their posters, or if they had to be unanimous in their message as a table. Table facilitators then fed back to the group about what was discussed and displayed their poster on a wall. Enabling participants to share and display their opinions is an effective facilitation method that can help build consensus in situations where opinions may be varied [27]. Hence, for the goals of the Ideas Café (Table 1), this methodology was an appropriate choice.

\section{Findings}

\subsection{Spectrum Line}

The lines were collected and the data converted into spreadsheet data by measuring the distance from each point to the left side of the line in Photoshop. Statistical differences between the two lines were analyzed using the Wilcoxon Signed Rank Test (Tables 4 and 5). The data from the lines was continuous, there were related groups between the two tests and distributions were verified from symmetry using boxplots.

Table 4. Descriptive statistics for the spectrum line

\begin{tabular}{l|l|l|l|l|l}
\hline Line & $\mathrm{N}$ & Mean & $25^{\text {th }}$ percentile & $50^{\text {th }}$ percentile & $75^{\text {th }}$ percentile \\
\hline First & 28 & 7694 & 6193 & 7780 & 9473 \\
\hline Second & 28 & 7902 & 5913 & 8861 & 9629 \\
\hline
\end{tabular}

Table 4 suggested that participants were in favor of driverless cars (on both lines), with the mean and 50th percentile being placed towards the more positive response on the spectrum line. However, the $\mathrm{Z}$ value $(-0.581)$ in Table 5 told us that there was no significant difference between the two lines.

In addition to capturing attitudes and perceptions, the activity helped participants feel settled and comfortable. Second, it provided the event with a strong user centric focus by enabling participants to easily share and publicly display their views, setting the precedent for participants to be more willing to share their opinions.

\subsection{Table Sessions}

All participant comments were transcribed into NVivo 11.4.3 (Mac). Qualitatively, a thematic analysis as part of grounded theory was run. The authors ran multiple cycles in order to mitigate the effects of subjectivity in the coding. The open ended qualitative format enabled us to collect rich data and find underlying themes that would not have 
Table 5. Wilcoxon signed ranks test for the spectrum line

\begin{tabular}{l|l|l|l}
\hline Second Line - First Line & $\mathrm{N}$ & Mean rank & Sum of ranks \\
\hline Negative ranks & 13 & 13.65 & 177.50 \\
\hline Positive ranks & 15 & 15.23 & 228.50 \\
\hline Z & -0.581 & \\
\hline Asymp. sig. (2 tailed) & 0.561 & \\
\hline
\end{tabular}

been possible with just a quantitative approach. Quantitatively, references to each code were summed for comparison. Sources were the number of unique participants, references were all instances of a code (to distinguish single participants who mentioned a code multiple times).

The codes were organized using structural coding according to the 3 table topics chosen (Tables 6, 7 and 8).

Table 6. Coding analysis for the theme: trust in technology

\begin{tabular}{l|l|c}
\hline Code & Sources & References \\
\hline Capabilities of vehicle & 7 & 9 \\
\hline Reliability & 7 & 10 \\
\hline Vehicle brand matters & 6 & 9 \\
\hline Coexistence of traditional and self-driving vehicles & 5 & 5 \\
\hline Aesthetics & 4 & 4 \\
\hline Service and maintenance & 4 & 5 \\
\hline Driving style & 4 & 4 \\
\hline Cost & 3 & 3 \\
\hline Nothing can stop driverless tech arriving & 3 & 3 \\
\hline
\end{tabular}

The capabilities and reliability of the vehicle were the key concerns. Participants appeared to draw on their previous experience with computers in general, "Computer systems are not all they should be" (P15) and "Technology can go wrong, it can do a lot of damage" (P7) when communicating their opinions on driverless vehicles; consistent with findings which suggest previous experience is a key aspect of trust [28, 29].

Both the capabilities and reliability featured with equal importance in participant responses (both with seven participants each). Some participants named specific issues, "can it be trusted with speed limits?" or "Can it be trusted with last minute changes?" (P25). The authors would suggest that the Reliability and Capability codes can be categorized under the theme of safety. Hence, ensuring the public are content and convinced with the safety of the technology would appear to develop trust in the users.

Participants also voiced concerns for how autonomous vehicles will work with existing vehicles on the road. All comments in this code were written as questions, suggesting research can do a better job of communicating the potential solutions to these trust issues. Consequently, what should be done to communicate the capabilities and reliability of the vehicle? Statistically proving the technology's safety is one 
possibility. However, it has been found that this would be impractical, requiring hundreds of millions of miles of testing to prove their safety [30]. With capability and reliability being key findings for the adoption of autonomous vehicles, it warrants further research as to how this can be communicated with the user.

Table 7. Coding analysis for the theme: data and privacy

\begin{tabular}{l|c|l}
\hline Code & Sources & References \\
\hline Safety risk & 11 & 16 \\
\hline Customizable privacy & 8 & 10 \\
\hline Acceptance that data is shared & 6 & 6 \\
\hline Not concerned & 6 & 8 \\
\hline Differential privacy & 5 & 5 \\
\hline Unaware of sharing & 5 & 5 \\
\hline Targeted advertising & 4 & 4 \\
\hline Data storage & 3 & 3 \\
\hline Reasons why & 3 & 4 \\
\hline
\end{tabular}

Hacking featured multiple times in the safety risk code, "Could they be hacked?" (P14), "Potential for hacking" (P2), and "Self-driving cars are open to cyber security threats, more susceptible to terrorism" (P34). From the table discussions, this appeared to be detrimental to trust with the participants.

Some participants were aware of the benefits of data sharing in creating more reliable and better supported systems, for example, "Good thing, data will help the technology work more efficiently" (P1), "Information ...communication are necessary to enhance the quality and reliability for self-driving vehicle" (P19). Some participants also remarked, "I accept that most of my data is already out there, especially my location at any given time" (P1) and "We already share our data" (P18).

The results would suggest that there was no general consensus on data sharing. One solution proposed by participants that appeared to be able to satisfy all viewpoints was the idea of differential or customizable privacy, giving control to the driver over what information was shared. However, this would need further research to determine which pieces of information would be deemed critical and non-customizable.

Differential privacy is a new techniques that allow analysis of data collected from personal devices whilst removing all personally identifying information from the data [31]. Though no participant specifically named this technique, it was evident from their responses that this could be an amicable solution.

Accessibility issues were voiced by participants, concerned with how the technology interacts with people, for example, "Would the technology be too complicated for the average person?" (P25) and "Control for all people, not just the technologist" (P8). Participants were able to describe methods akin to those used in participatory design that could solve this, "Technology can be trustworthy, but it needs to start from a certain group of people (and not engineers) to assure that it's working" (P5) and "Involve public, i.e. buses and taxis should be involved" (P23). 
Table 8. Coding analysis for the theme: societal impact

\begin{tabular}{l|l|l}
\hline Code & Sources & References \\
\hline Accessibility issues & 7 & 10 \\
\hline Involve people in the design & 7 & 9 \\
\hline Legal, regulatory & 7 & 9 \\
\hline Concerns with no driver & 6 & 9 \\
\hline Infrastructure & 4 & 4 \\
\hline Adoption of technology & 3 & 4 \\
\hline Age issues & 3 & 4 \\
\hline Pedestrians & 3 & 6 \\
\hline Physical privacy & 3 & 4 \\
\hline
\end{tabular}

Communication was raised as an important factor in the adoption of the new technology, "Unbiased communication towards building trust" (P23), "Use of language- explain why needed" (P16). Studies have found that in any change process, good communication and the language used to deliver the message is critical to the success of the change initiative [32]. It also highlights the importance of events like Ideas Cafes as a tool for communicating with the public, and how participants perceive good communication as a key part of building trust. These findings suggest that the issue of building trust is not a technical problem, but one that is based primarily on good communication with the public.

The next major theme was the legal and regulatory impact. For example, "Who is liable? [in a crash]" (P25), "Needs to be regulated, legal and ethical" (P10). These appeared to stem from the lack of human driver, leading to accessibility concerns. For example, a few elderly participants remarked, "who will take luggage and help visually impaired to reception desk (e.g. In a taxi)" (P6), "remember the human element of taxi drivers" (P8), "Trust late at night, no bus or taxi driver, not good for reassurance" (P6). These issues are present regardless of the maturity of autonomous technology and raises questions as to what kind of solution can provide the same level of tertiary services (such as help with luggage) as a human driver. The results make it evident that though increased accessibility is touted as a beneficial feature to older users who will be able to maintain their independent travel, there are other aspects which may be more detrimental to the vehicle user experience to older drivers.

\subsection{Key Emergent Themes}

The authors then reviewed the codes and reorganized the data from the structural coding of the table sessions. The result is presented below in Fig. 1. The results suggest that the effects of autonomous vehicles on society and policy are the biggest factor determining trust. This is followed closely by data and privacy issues. To the authors, this suggests that until the impact of the technology on broader society and privacy is understood, trust may struggle to be established. Much of current work is focused on establishing trust through interface design [33, 34], which is important, but only a part of the issue of trust. The authors were only able to find one model of trust in 
autonomous vehicles that draws specific attention to the need to consider the design of society to establish trust in the technology [35].

\begin{tabular}{|l|}
\hline \multicolumn{1}{|c|}{ Society and Policy } \\
\hline - Vehicle Brand \\
- Coexistence of \\
traditional and \\
self driving \\
vehicles \\
- Service and \\
Maintenance \\
- Legal, REgulatory \\
- Infrastructure \\
- Adoption of \\
Technology \\
- Physical Privacy \\
- Concerns with no \\
driver \\
\hline
\end{tabular}
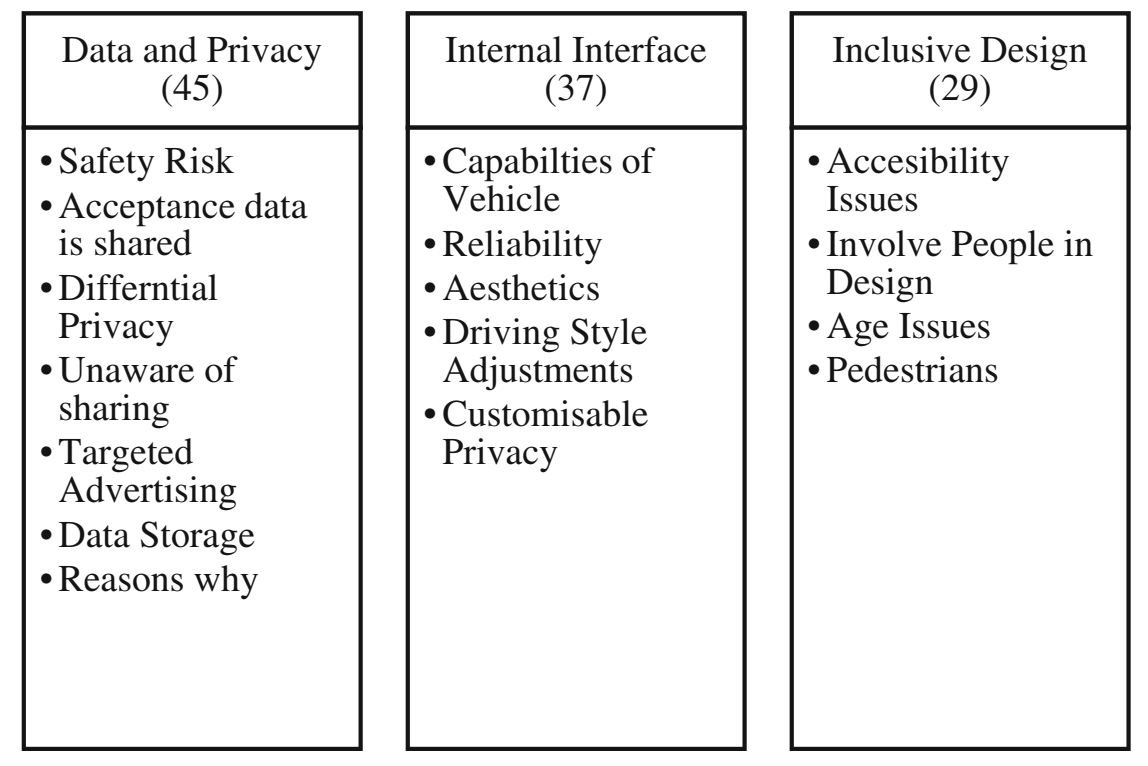

Fig. 1. Coding themes related to trust in autonomous vehicles

The codes relating to the internal interface are important for the authors' research and will be addressed in future work.

\section{Limitations}

A limitation of the spectrum line is the possibility that the question "Do you think driverless cars are a good idea?" is leading. A more appropriately designed question would have been "What do you think about driverless cars?".

While the lineup was a useful tool as an energizer for participants, it had limited value to the results because of time constraints on the event day and difficulties in capturing the result. The authors recommend that future events could plan for longer time to be given to the exercise, perhaps capturing the positions with a panoramic photograph.

The table sessions provided the most significant proportion of the data collected from the event. The Ideas Café format allowed participants freedom to discuss and approach the topic as they saw fit. This is both an advantage and disadvantage; while this allowed the results to cover a broad spectrum of topics, it also meant that no specific topic could be explored in particular depth.

This alludes to one of the limitations of the Ideas Café in that it did not audio record the sessions, this placed greater stress on the expert facilitators on taking detailed notes and encouraging participants to write all their thoughts and comments. Audio recording the tables would have been beneficial, but difficult to analyze given the number of participants on each table. 


\section{Conclusion}

This paper set out to understand the factors affecting trust in autonomous vehicles and has provided thematically derived recommendations for future research. The Ideas Café is unique in providing an informal setting to deploy a variety of different engagement tools with the express purpose of creating a two way flow of information from the expert to the user. Other methods like Charrettes [36] and Focus Groups [37] appear to be similar, but place far greater emphasis on defining a specific topic to solve. The Ideas Café takes a more informal, open approach, providing participants with a relaxed café style environment with cake and tea and more focus on two way communication. The highly positive feedback from participants, as well as the fact that all participants were happy to be invited back to take part in future research, were endorsements of the method. This means the authors are now able to access a large pool of participants for future research in the area. This was an unexpected but useful outcome.

The authors have demonstrated how it is possible to gather perceptions of trust by engaging with the public using an Ideas Café. A better understanding of the factors that must be addressed for users to trust the autonomous vehicle was gained. Specifically, the importance of designing for the technology's integration with society was highlighted. The value of this work is a recognition that this broader societal level design is as important as the vehicle design itself. The authors aim to ensure that the issues highlighted by the event will be taken forward into future research.

\section{References}

1. Petts, J.: Public engagement to build trust: false hopes? J. Risk Res. 11, 821-835 (2008)

2. Yang, K., Pandey, S.K.: Further dissecting the black box of citizen participation: when does citizen involvement lead to good outcomes? Public Adm. Rev. 71, 880-892 (2011)

3. Irwin, A.: Constructing the scientific citizen: science and democracy in the biosciences. Public Underst. Sci. 10, 1-18 (2001)

4. Inayatullah, S.: Six pillars: futures thinking for transforming. Foresight 10, 4-21 (2008)

5. Varum, C.A., Melo, C.: Directions in scenario planning literature-a review of the past decades. Futures 42, 355-369 (2010)

6. Held, D.: Democracy and the global order (1995)

7. Cooper, T.L., Bryer, T.A., Meek, J.W.: Citizen-centered collaborative public management. Public Adm. Rev. 66, 76-88 (2006)

8. Hoff, K.A., Bashir, M.: Trust in automation integrating empirical evidence on factors that influence trust. Hum. Factors J. Hum. Factors Ergon. Soc. 57, 407-434 (2015)

9. Cranor, L.F.: A framework for reasoning about the human in the loop. In: UPSEC, vol. 8, pp. 1-15 (2008)

10. Strand, N., Nilsson, J., Karlsson, I.M., Nilsson, L.: Semi-automated versus highly automated driving in critical situations caused by automation failures. Veh. Autom. Driver Behav. 27, 218-228 (2014) 
11. Khastgir S., Birrell S., Dhadyalla G., Jennings, P.: Calibrating trust to increase the use of automated systems in a vehicle. In: Stanton N., Landry S., Di Bucchianico, G., Vallicelli, A. (eds) Advances in Human Aspects of Transportation. Advances in Intelligent Systems and Computing, vol. 484, pp. 535-546. Springer, Cham (2017). https://doi.org/10.1007/978-3319-41682-3_45

12. Muir, B.M.: Trust between humans and machines, and the design of decision aids. Int. J. Man Mach. Stud. 27, 527-539 (1987)

13. Parasuraman, R., Manzey, D.H.: Complacency and bias in human use of automation: an attentional integration. Hum. Factors J. Hum. Factors Ergon. Soc. 52, 381-410 (2010)

14. Dzindolet, M.T., Peterson, S.A., Pomranky, R.A., Pierce, L.G., Beck, H.P.: The role of trust in automation reliance. Int. J. Hum. Comput. Stud. 58, 697-718 (2003)

15. Jian, J.-Y., Bisantz, A.M., Drury, C.G.: Foundations for an empirically determined scale of trust in automated systems. Int. J. Cogn. Ergon. 4, 53-71 (2000)

16. Spain, R.D., Bustamante, E.A., Bliss, J.P.: Towards an empirically developed scale for system trust: Take two. Hum. Factors Ergon. Soc. Annu. Meet. Proc. 52(19), 1335-1339 (2008)

17. Fallon, C.K., Bustamante, E.A., Ely, K.M., Bliss, J.P.: Improving user trust with a likelihood alarm display. Presented at the proceedings of the 1st international conference on augmented cognition, Las Vegas, NV (2005)

18. McCarley, J.S., Wiegmann, D.A., Wickens, C.D., Kramer, A.F.: Effects of age on utilization and perceived reliability of an automated decision-making aid for luggage screening. Proc. Hum. Factors Ergono. Soc. Annu. Meet. 47(3), 340-343 (2003)

19. Hedges, A., Sykes, W., Groom, C.: Extending working life: changing the culture. Qualitative research into effective messages (2009). Department for Work and Pensions

20. Mechanic, D., Meyer, S.: Concepts of trust among patients with serious illness. Soc. Sci. Med. 51, 657-668 (2000)

21. Muñoz-Leiva, F., Luque-Martínez, T., Sánchez-Fernández, J.: How to improve trust toward electronic banking. Online Inf. Rev. 34, 907-934 (2010)

22. Rowe, G., Frewer, L.J.: A typology of public engagement mechanisms. Sci. Technol. Hum. Values 30, 251-290 (2005)

23. Brown, J., Isaacs, N.M.: Hosting conversations that matter at the world cafe. Whole Syst. Assoc. 1, 1-20 (2002)

24. Berthet, E.T., Barnaud, C., Girard, N., Labatut, J., Martin, G.: How to foster agroecological innovations? A comparison of participatory design methods. J. Environ. Plan. Manag. 59, 280-301 (2016)

25. Berger, J., Fitzsimons, G.: Dogs on the street, pumas on your feet: how cues in the environment influence product evaluation and choice. J. Mark. Res. 45, 1-14 (2008)

26. Carlsson, A.M.: Assessment of chronic pain. I. Aspects of the reliability and validity of the visual analogue scale. Pain 16, 87-101 (1983)

27. Bryson, J.M., Anderson, S.R.: Applying large-group interaction methods in the planning and implementation of major change efforts. Public Adm. Rev. 60, 143-162 (2000)

28. Lee, J.D., See, K.A.: Trust in automation: designing for appropriate reliance. Hum. Factors J. Hum. Factors Ergon. Soc. 46, 50-80 (2004)

29. Teacy, W.L., Patel, J., Jennings, N.R., Luck, M.: Travos: trust and reputation in the context of inaccurate information sources. Auton. Agent. Multi-Agent Syst. 12, 183-198 (2006)

30. Kalra, N., Paddock, S.M.: Driving to safety: how many miles of driving would it take to demonstrate autonomous vehicle reliability? Transp. Res. Part A: Policy Pract. 94, 182-193 (2016)

31. Eigner, F., Maffei, M.: Differential privacy by typing in security protocols, pp. 272-286 (2013) 
32. Bordia, P., Hunt, E., Paulsen, N., Tourish, D., DiFonzo, N.: Uncertainty during organizational change: is it all about control? Eur. J. Work Organ. Psychol. 13, 345-365 (2004)

33. Choi, J.K., Ji, Y.G.: Investigating the importance of trust on adopting an autonomous vehicle. Int. J. Hum. Comput. Interact. 31, 692-702 (2015)

34. Koo, J., Kwac, J., Ju, W., Steinert, M., Leifer, L., Nass, C.: Why did my car just do that? Explaining semi-autonomous driving actions to improve driver understanding, trust, and performance. Int. J. Interact. Des. Manuf. 9, 269-275 (2014)

35. Schaefer, K.E., Chen, J.Y.C., Szalma, J.L., Hancock, P.A.: A meta-analysis of factors influencing the development of trust in automation. Hum. Factors J. Hum. Factors Ergon. Soc. 58, 377-400 (2016)

36. Gibson, G.E., Whittington, D.A.: Charrettes as a method for engaging industry in best practices research. J. Constr. Eng. Manag. 136, 66-75 (2010)

37. Asbury, J.-E.: Overview of focus group research. Qual. Health Res. 5, 414-420 (1995) 